\title{
Report on UQ Assessments to Support SESAME Wire-Wrapped Bundle Experiment
}

\section{Approved for public release.} Distribution is unlimited.

Marc-Olivier G. Delchini Emilian L. Popov

David W. Pointer

09/30/2017 


\section{DOCUMENT AVAILABILITY}

Reports produced after January 1, 1996, are generally available free via US Department of Energy (DOE) SciTech Connect.

Website: http://www.osti.gov/scitech/

Reports produced before January 1, 1996, may be purchased by members of the public from the following source:

National Technical Information Service

5285 Port Royal Road

Springfield, VA 22161

Telephone: 703-605-6000 (1-800-553-6847)

TDD: $703-487-4639$

Fax: 703-605-6900

E-mail: info@ntis.gov

Website: http://classic.ntis.gov/

Reports are available to DOE employees, DOE contractors, Energy Technology Data Exchange representatives, and International Nuclear Information System representatives from the following source:

Office of Scientific and Technical Information

PO Box 62

Oak Ridge, TN 37831

Telephone: $865-576-8401$

Fax: 865-576-5728

E-mail: report@osti.gov

Website: http://www.osti.gov/contact.html

This report was prepared as an account of work sponsored by an agency of the United States Government. Neither the United States Government nor any agency thereof, nor any of their employees, makes any warranty, express or implied, or assumes any legal liability or responsibility for the accuracy, completeness, or usefulness of any information, apparatus, product, or process disclosed, or represents that its use would not infringe privately owned rights. Reference herein to any specific commercial product, process, or service by trade name, trademark, manufacturer, or otherwise, does not necessarily constitute or imply its endorsement, recommendation, or favoring by the United States Government or any agency thereof. The views and opinions of authors expressed herein do not necessarily state or reflect those of the United States Government or any agency thereof. 
Nuclear and Reactor Safety Division

\section{REPORT ON UQ ASSESSMENTS TO SUPPORT SESAME WIRE-WRAPPED BUNDLE EXPERIMENT}

Marc-Olivier G. Delchini, Emilian L. Popov and David W. Pointer

Date Published: September 2017

Prepared by

OAK RIDGE NATIONAL LABORATORY

Oak Ridge, TN 37831-6283

managed by

UT-Battelle, LLC

for the

US DEPARTMENT OF ENERGY

under contract DE-AC05-00OR22725 



\section{CONTENTS}

LIST OF FIGURES $\ldots \ldots \ldots \ldots \ldots \ldots \ldots \ldots \ldots \ldots$

LIST OF TABLES $\ldots \ldots \ldots \ldots \ldots \ldots \ldots \ldots \ldots$ vii

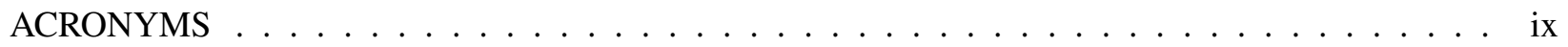

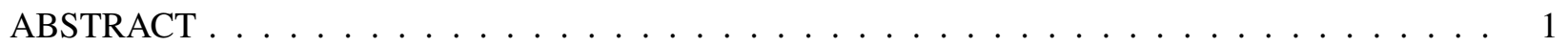

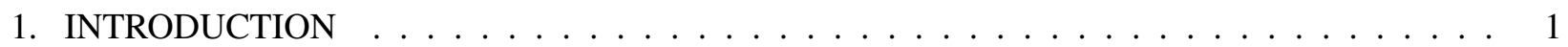

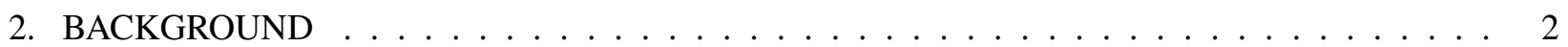

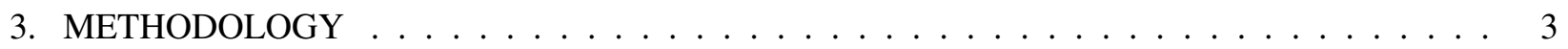

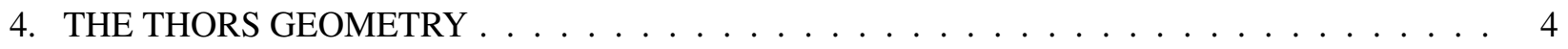

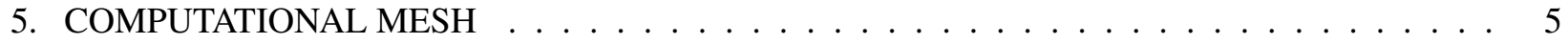

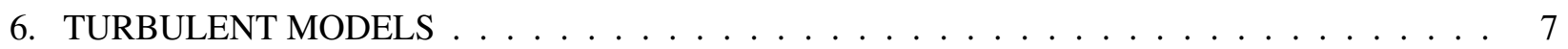

7. THE SINGLE-PIN GEOMETRY: NUMERICAL RESULTS AND SENSITIVITY ANALYSIS . . 8

7.1 Boundary Conditions, Initial Conditions and Workflow . . . . . . . . . . . . . . 8

7.2 Sensitivity Analysis of the Pressure Drop to the Mesh Density . . . . . . . . . . . . . 9

7.3 Sensitivity Analysis of the Pressure Drop to Turbulent Models . . . . . . . . . . . . . . 10

7.4 Sensitivity Analysis of Pressure Drop to Mesh Type . . . . . . . . . . . . . . . . . . 10

7.5 Sensitivity Analysis of the Pressure Drop to the Number of Wire Pitch . . . . . . . . . . 11

7.6 Comparison of STAR-CCM+ Pressure Drops with Experimental and Correlation Data . . . 11

8. THE 7-PIN WIRE-WRAPPED GEOMETRY: NUMERICAL RESULTS AND SENSITIVITY

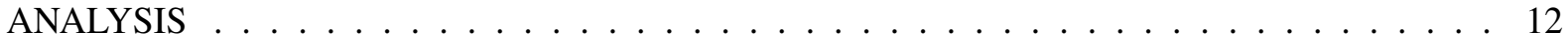

8.1 Boundary Conditions, Initial Conditions and Workflow _ . . . . . . . . . . . . . 12

8.2 Sensitivity of the Pressure Drops to the Open- and Closed-gap Approaches . . . . . . . . . 12

9. THE 19-PIN WIRE-WRAPPED GEOMETRY: NUMERICAL RESULTS AND

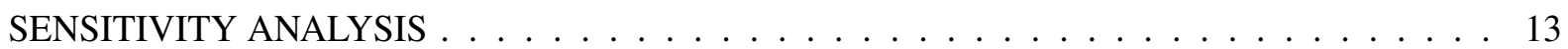

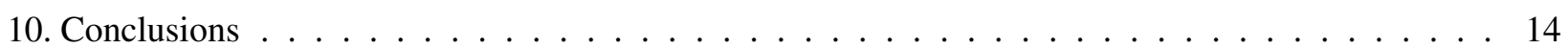

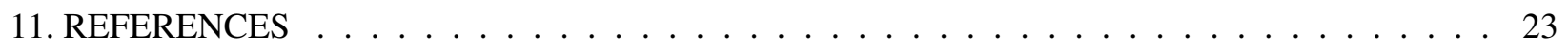





\section{LIST OF FIGURES}

1 A wire-wrapped pin bundle geometry ( $D_{w}$ is the wire diameter, $\mathrm{D}$ is the pin diameter, $\mathrm{H}$ is the lead height, $\mathrm{P}$ is the pin pitch, and $\mathrm{W}$ is the pitch to the wall) . . . . . . . . . 3

2 closed-gap approach versus open-gap approach for the 7-pin bundle geometry. . . . . . . 6

3 closed-gap approach versus open-gap approach for the single-pin bundle geometry. . . . . . 7

4 Hex and poly meshes of the single-pin geometry for closed-gap approach and open-gap

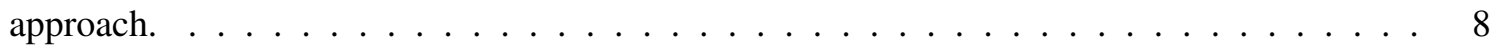

5 closed-gap approach versus open-gap approach for the 7-pin bundle geometry. . . . . . . . 9

6 Comparison of STAR-CCM+ pressure drops between experimental and correlation data with the polyhedral mesh and open-gap approach for the single-pin geometry. . . . . . . . 15

7 Comparison of STAR-CCM+ pressure drops between experimental and correlation data with the polyhedral mesh and closed-gap approach for single-pin geometry. . . . . . . . . 16

8 Comparison of STAR-CCM+ pressure drops between experimental and correlation data with the hexahedral mesh and closed-gap approach for the single-pin geometry. . . . . . . . 17

9 Comparison of STAR-CCM+ pressure drops between experimental and correlation data with the hexahedral mesh and open-gap approach for the single-pin geometry. . . . . . . . 18

10 Comparison of STAR-CCM+ pressure drop results between experimental and correlation data for the 7-pin geometry with closed-gap approach and polyhedral mesh. . . . . . . . . . 19

11 Comparison of STAR-CCM+ pressure drop results between experimental and correlation data for the 7-pin geometry with open-gap approach and polyhedral mesh . . . . . . . . . . 20

12 Comparison of STAR-CCM+ pressure drop results between experimental and correlation data for the 19-pin geometry with open-gap approach and polyhedral mesh. . . . . . . . . . 21 



\section{LIST OF TABLES}

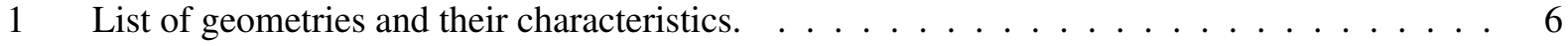

2 Sensitivity of pressure drop to polyhedral mesh density for the single-pin geometry . . . . 9

3 Sensitivity of pressure drop to turbulent models for the single-pin geometry $\ldots \ldots \ldots$

4 Sensitivity of pressure drop to mesh type (hex and poly) for the single-pin geometry . . . . . 10

5 Sensitivity of pressure drop to number of lead wire-pitch for the single-pin geometry $\ldots \ldots 11$ 



\section{ACRONYMS}

ORNL Oak Ridge National Laboratory

CAD computer-aided design

CFD computational fluid dynamics

CPU computational processor unit

FFM fuel failure mockup

LES large eddy simulation

LMFBR liquid metal fast breeder reactor

LMR liquid metal reactor

RANS Reynolds Averaged Navier Stokes

THORS thermal- hydraulic out-of-reactor safety 



\section{ACKNOWLEDGMENTS}

This work was supported by the Nuclear Energy Advanced Modeling and Simulation (NEAMS) program under the auspices of the US Department of Energy, Office of Nuclear Energy. 



\begin{abstract}
This work assesses the influence of assumptions made when generating a mesh of a wire-wrapped geometry. The contact region between a wire and its adjacent pin is commonly modeled by either embedding the wire to the adjacent pin or trimming the wire so that a gap separates the wire from its adjacent pin. These models are referred to as closed-gap and open-gap approaches herein and are applied to two geometries. The first geometry consists of a single pin wire-wrapped subchannel. A polyhedral mesh and a hexahedral mesh are generated. The second and third geometries are a 7- and 19-pin wire-wrapped bundles meshed with polyhedral elements only. Pressure drops are obtained with the STAR-CCM+ computational fluid dynamic (CFD) package. Sensitivity analyses of the mesh density, the mesh type, and the turbulent models are performed. Numerical results show that the best matches to the experimental data and to the Cheng-Todreas correlation are obtained with the combination of a hexahedral mesh, the shear stress transport (SST) turbulent model, and the open-gap approach. In the case of the 7-pin geometry, the best results are obtained with the open-gap approach and the SST turbulent model. The 19-pin geometry yields contradictory results to the 7-pin geometry results, and thus will require further investigations.
\end{abstract}

\title{
1. INTRODUCTION
}

Liquid metal reactors have gained interest in the past years, as they offer many benefits over the more common pressurized and boiling water reactor designs. They are in principle easier to operate and to maintain and have better inherent safety, as they operate at atmospheric pressure conditions. The high boiling temperature of liquid metal compared to water allows for a higher operating temperature, yielding higher thermal efficiency and more compact core design through the use of narrower subchannels. They also represent an essential technology for the implementation of a multi-stage fuel cycle that should allow for a more efficient use of natural resources while reducing challenges associated with long-term used fuel repository.

In response to the objectives outlined by the Advanced Fuel Cycle Initiative, an effort has been initiated to assess the applicability of a variety of thermal hydraulic analysis methods to predict heat transfer and fluid dynamic phenomena involved in wire-wrapped bundle assemblies. For example, an objective is to predict and optimize the mixing behavior to reduce hot channel factors and consequently to allow operation at higher temperatures. 


\section{BACKGROUND}

One unique characteristic of liquid metal reactors lies in the design of bundles made of long, thin, wire-wrapped rods. The helical-type wire spaces were originally designed to avoid collision between adjacent rods, but they are also known to enhance flow mixing by inducing secondary and cross flows from inner to outer subchannel, leading to a more uniform coolant temperature throughout the core. However, the helical-type wire spaces also promote undesirable phenomena such as an increase in the pressure drop. Another characteristics of a wire-wrapped bundle geometry is the numerous contact lines and points between pins and wires that make the geometry complex to generate, and the computational mesh difficult to create. In addition to the complexity of the geometry, the flow is known to be turbulent with Reynolds numbers in the range of $40 \times 10^{3}$ to $65 \times 10^{3}$. (The Reynolds number is herein defined based on a hydraulic diameter of a pin.) Because of the complex flow features in wire-wrapped bundle and the complex associated geometry, experimental and numerical studies are crucial for understanding the flow characteristics in subchannels of wire-wrapped bundles and for determining design limit parameters.

Many experimental studies have been conducted to investigate the thermal-hydraulics of wire-wrapped fuel bundles. These experimental efforts provided for the development of pressure drop correlations specific to the wire-wrapped bundles and the function of geometric parameters. Pressure drops in such complex geometries cannot be predicted by a simple equivalent diameter technique and thus require special efforts. Rehme [13] proposed correlations to predict the friction factor in a 7-pin bundle with a helical wire spacer based on water experiments. Noverdstern [10] developed a semi-empirical model to predict pressure loss in bundles with wire spacers. Cheng and Todreas [15] developed friction factor correlations in wire-wrapped bundles and accounted for all flow regimes and geometric effects. Recently, Chen et al. [5] assessed the accuracy of five published pressure drop correlations against eighty pressure drop datasets.

Early numerical studies of wire-wrapped bundles were performed with subchannel analysis codes such as COBRA [14], SABRE [8] and ASFRE [9] because of limited computing resources. Subchannel codes solve for subchannel-averaged quantities and cannot resolve locally developed flow phenomena. Instead, subchannel codes rely on mixing source terms function of correlations derived from experiments to model turbulent effects and predict pressure drops.

With the recent growth in high-performance computing capabilities, numerical simulations of flow in wire-wrapped bundles were carried out using CFD open-source and commercial packages $[11,2,12,6]$. These studies allow for more detailed numerical investigations of turbulent flow in wire-wrapped bundles such as secondary and cross flow triggered by the helical-type wire spacer. They also allow for numerical prediction of the pressure drop for comparison against correlations. Reynolds-Averaged Navier-Stokes (RANS)-based simulations with the STAR-CCM+ CFD package are commonly used to simulate flows in wire-wrapped bundle geometries, as they require less computing resources than large eddy simulations (LES). 


\section{METHODOLOGY}

RANS simulations are carried out in this study with the STAR-CCM+ CFD commercial package. STAR-CCM+ is a finite volume formulation code used to simulate compressible and incompressible flows, as well as heat transfer. It supports hexahedral and polyhedral meshes but favors the use of the later, as it greatly simplifies the generation of the computational meshes for complex geometries. STAR-CCM+ implements a variety of RANS model and boundary conditions.

CFD packages require generation of computational mesh to simulate flow behaviors in a given geometry. In a wire-wrapped bundle geometry, generation of a computational domain is challenging. The wire wraps are helically wound along each pin and are spot-welded at the top and bottom of the pin, but they are not attached to the entire length of the pin (Fig. 1). This design creates multiples contact points and lines

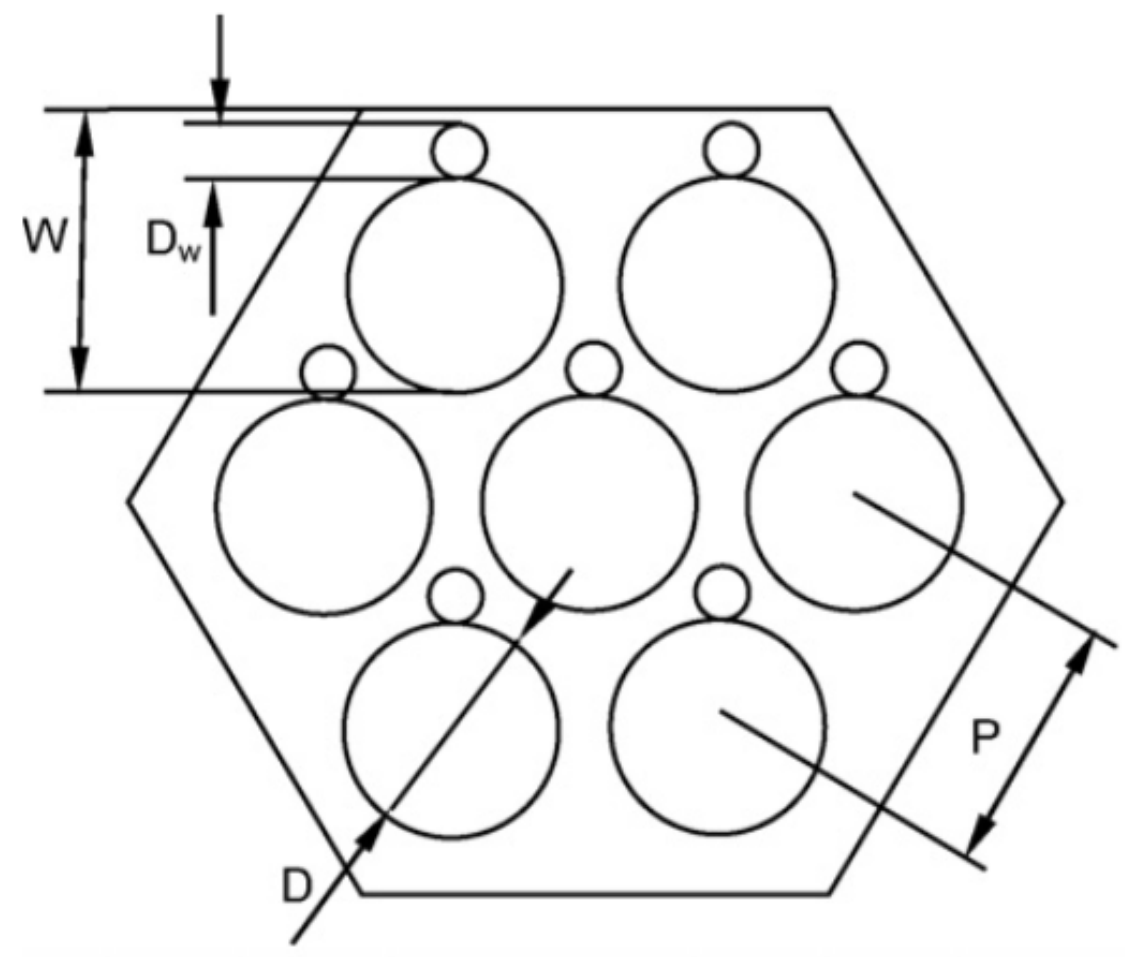

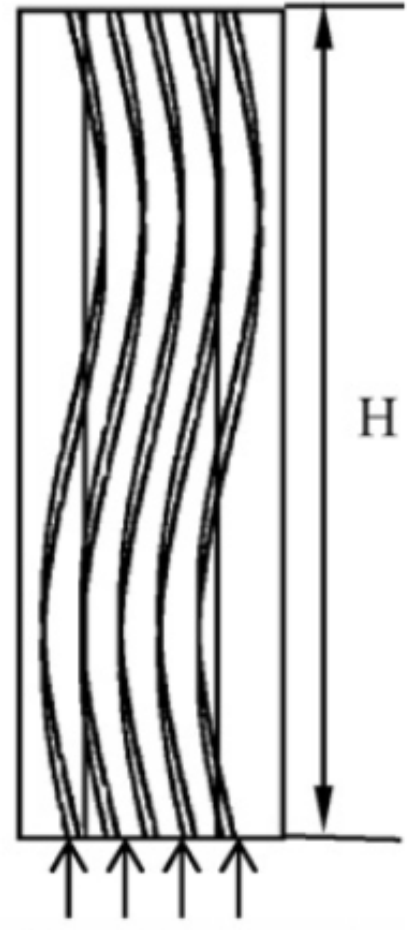

Flow Direction

Figure 1. A wire-wrapped pin bundle geometry $\left(D_{w}\right.$ is the wire diameter, $\mathbf{D}$ is the pin diameter, $\mathbf{H}$ is the lead height, $P$ is the pin pitch, and $W$ is the pitch to the wall).

between the wire and the pin, and between the wire and its adjacent pin, that require approximations or models to generate a computational geometry and mesh. Furthermore, the

wire-wrapped-bundle-length-to-subchannel-hydraulic diameter ratio is typically on the order of 360, which increases the number of elements required to obtain a converged numerical solution by a considerable amount.

This report investigates the sensitivity of the pressure drop to the modeling of the wire-adjacent pin region for wire-wrapped bundles. The objective is to determine whether the model chosen for the wire and the 
adjacent pin has a significant effect on the pressure drop predicted by RANS simulations obtained with the STAR-CCM+ CFD code. Each approach involves various approximations when generating the geometry to be discussed. The case study in this report is the Thermal-Hydraulic Out-of-Reactor Safety (THORS) facility built at Oak Ridge National Laboratory (ORNL) which made pressure drop experimental data available. Single-pin, 7-pin, and 19-pin wire-wrapped bundles are investigated. Numerical results are compared against correlations and experimental data to determine the best approach to maximize the flow resolution, to accurately predict the pressure drop, to minimize the computing time, and to keep the mesh size within acceptable range.

\section{THE THORS GEOMETRY}

The Fuel Failure Mockup (FFM) facility and THORS were large thermal hydraulic liquid metal (sodium) loops built at Oak Ridge National Laboratory (ORNL) in 1970, with testing started in 1971. The final tests took place in December of 1984, the program was closed in 1985, and the facilities were decommissioned. The purpose of these tests was to support the Liquid Metal Fast Breeder Reactor (LMFBR) Program by acquiring thermal hydraulic data on liquid metal.

During the 15 years of FFM and THORS operation, 11 bundles were designed, 7 of which were built and tested in 14 different configurations. The initial charter of the program was to investigate bundle blockages, but the emphasis shifted to transient tests, sodium boiling, and dryout. All reports and data from the complete FFM and THORS programs are available from ORNL [3]. These data can be used to support future liquid metal reactor (LMR) programs and to benchmark liquid metal thermal hydraulic computer models.

Due to data classification (Applied Technology), this report does not include any specific sizes of the modeled geometry, but it provides details on generated computational meshes. Three geometries with THORS-designed parameters are considered in this report: (1) a wire-wrapped pin in a triangular matrix, referred to as single-pin geometry, (2) a 7-pin wire-wrapped bundle geometry, or in short, 7-pin bundle geometry, and (3) a 19-pin wire wrapped bundle geometry (19-pin bundle geometry). Each geometry is created with a CAD software and meshed with either HEXPRESS or the STAR-CCM+ built-in meshing features. Further details relevant to the CAD models and the meshes are provided in Section 6. 


\section{COMPUTATIONAL MESH}

In the RANS studies, the geometry is preferably meshed with polyhedral elements over hexahedral elements since polyhedral meshes yield better convergence behavior. Moreover, there seems to be a discrepancy in the literature on the modeling of the region between the wire and the adjacent pins. This discrepancy arises from the complexity to mesh the contact point between the wire and the adjacent pin. Two approaches are commonly used in the literature. For example, in Pointer et al. [12], the wire is trimmed near the adjacent pin (see Fig. 5 of [12] or Fig. 4b herein), creating a gap region between the wire and the adjacent pin. With this approach, the contact point is "replaced" by a gap. Another approach used in Jeong et al. [7] consists of embedding the wire to the adjacent pin (see Fig. 3 of [7] or Fig. 5a herein), replacing the contact point by a line that can be easily meshed. These two approaches seem to lead to reasonable prediction of pressure drops when compared against correlation and experimental data. However, there is a lack of comparative study between the two approaches, i.e., open-gap vs closed-gap, for wire-wrapped bundles.

Two options are proposed to model the contact point when creating the geometry with a CAD package and to facilitate the meshing process:

- closed-gap case: the geometry is locally modified in the contact region between the wire and the pin by either reducing the pitch or increasing the wire diameter so that the wire and the pin surfaces overlap. With this option, the pin diameter is not altered, but the hydraulic diameter is modified since a small portion of the wire is now embedded in the pin.

- open-gap case: the contact point is replaced by a gap between the wire and the neighbor pin. This is achieved by either trimming the wire or reducing the wire's diameter. The downside of this approach is that a very fine mesh is required near the gap region to capture the local flow features, leading to a dramatic increase of the number of elements. It is anticipated that the smaller the gap, the larger the number of elements in the mesh.

For both options, modifications to the original geometry are considered acceptable as long as the mismatch remains within $5 \%$ of the wire diameter, which corresponds to the fabrication tolerance. It is anticipated that by modifying the geometry locally in the contact region, the flow behavior and pressure drop will also be altered. One objective of this study is to identify which of these options better matches the experimental pressure drops and correlation data within reasonable tolerance and computational processor unit (CPU) time.

Illustrations of the closed-gap and open-gap approaches are presented in Fig. 2 for the 7-pin bundle geometry and in Fig. 3 for the single-pin geometry. The 19-pin geometry is not shown here but was generated using the same parameters as for the 7-pin geometry. The red domain corresponds to the computational domain of interest, i.e.: the coolant. In Fig. 2, horizontal cross sections of the 7-pin bundle geometry along the stream-wise direction are presented to illustrate the closed- and open-gap cases. In Fig. 2a, the wire is in contact with pin 1 and the adjacent pin which corresponds to the closed-gap configuration. In Fig. 2b, the open-gap configuration is illustrated. The wire is only attached to pin 1, and a gap is present between the wire and the adjacent pin. The same two configurations were also used to generate the single-pin geometries, as shown in Fig. 3. Characteristics of the meshes generated for this work are presented in Table 1. Polyhedral (poly) and hexahedral (hex) elements are used to mesh the single-pin geometry modeled with open- and closed-gap approaches. The hexahedral meshes are generated 


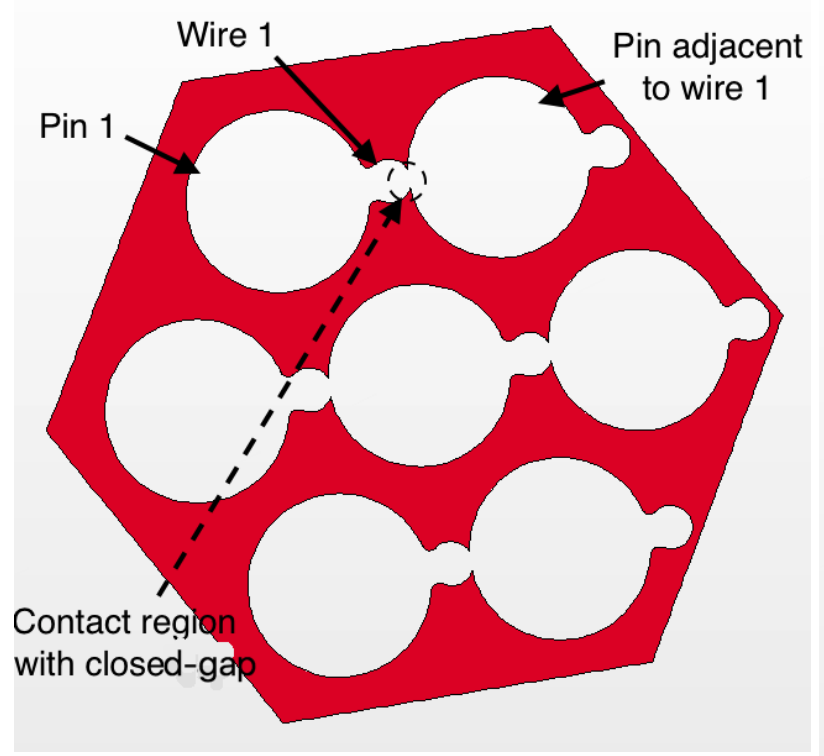

(a) closed-gap approach.

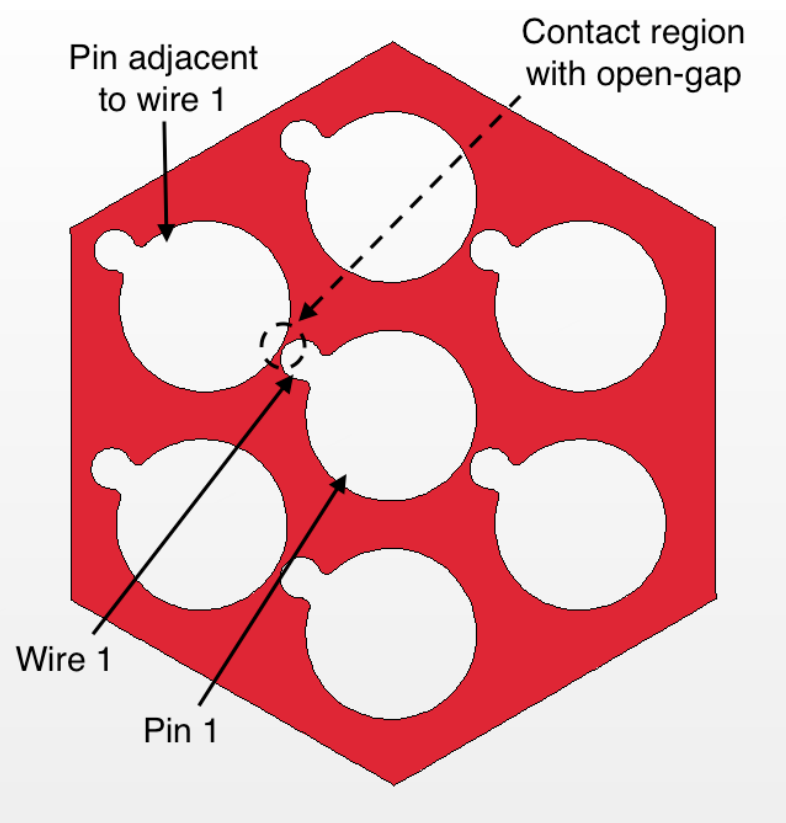

(b) Open-gap approach.

Figure 2. closed-gap approach versus open-gap approach for the 7-pin bundle geometry.

with the HEXPRESS commercial package, whereas the polyhedral meshes are created with the STAR-CCM+ built-in mesher. A vertical cross section of the meshed single-pin geometry is shown in Fig. 4 for open- and closed-gap approaches. Table 1 shows that the mesh density of the single-pin hex mesh with an open-gap model is between 3 to 6 times higher than the other meshes for the same geometry. This is due to the open-gap approach that requires a small region between the wire and the adjacent pin to be meshed. The poly and hex meshes of the single-pin geometry will be imported in STAR-CCM+ to perform a sensitivity analysis of the pressure drop to the mesh type. The 7- and 19-pin geometries are only meshed with polyhedral elements, and a mesh sample of a horizontal cross section is provided in Fig. 5 for the 7-pin geometry.

Table 1. List of geometries and their characteristics.

\begin{tabular}{llllll}
\hline geometry & contact & $\begin{array}{l}\text { element } \\
\text { type }\end{array}$ & $\begin{array}{l}\text { number of } \\
\text { elements }\end{array}$ & $\begin{array}{l}\text { geometry } \\
\text { ID }\end{array}$ & figure \\
single-pin & closed-gap (Fig. 3a) & hexahedral & $299 \mathrm{k}$ & sha & Fig. 4a \\
single-pin & open-gap (Fig. 3b) & hexahedral & $2.7 \mathrm{M}$ & shb & Fig. 4b \\
single-pin & closed-gap (Fig. 3a) & polyhedral & $796 \mathrm{k}$ & spa & Fig. 4c \\
single-pin & open-gap (Fig. 3b) & polyhedral & $287 \mathrm{k}$ & spb & Fig. 4d \\
7-pin & closed-gap (Fig. 2a) & polyhedral & $9.7 \mathrm{M}$ & $7 \mathrm{a}$ & Fig. 5a \\
7-pin & open-gap (Fig. 2b) & polyhedral & $13.6 \mathrm{M}$ & $7 \mathrm{~b}$ & Fig. 5b \\
19-pin & open-gap & polyhedral & $12.9 \mathrm{M}$ & $7 \mathrm{~b}$ & N/A \\
\hline
\end{tabular}




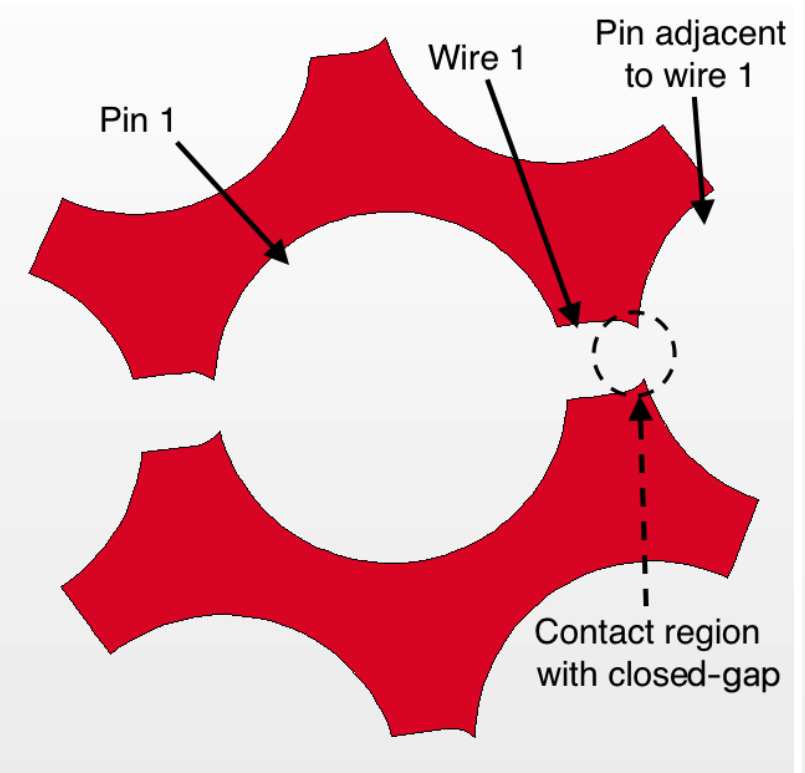

(a) closed-gap appraoch.

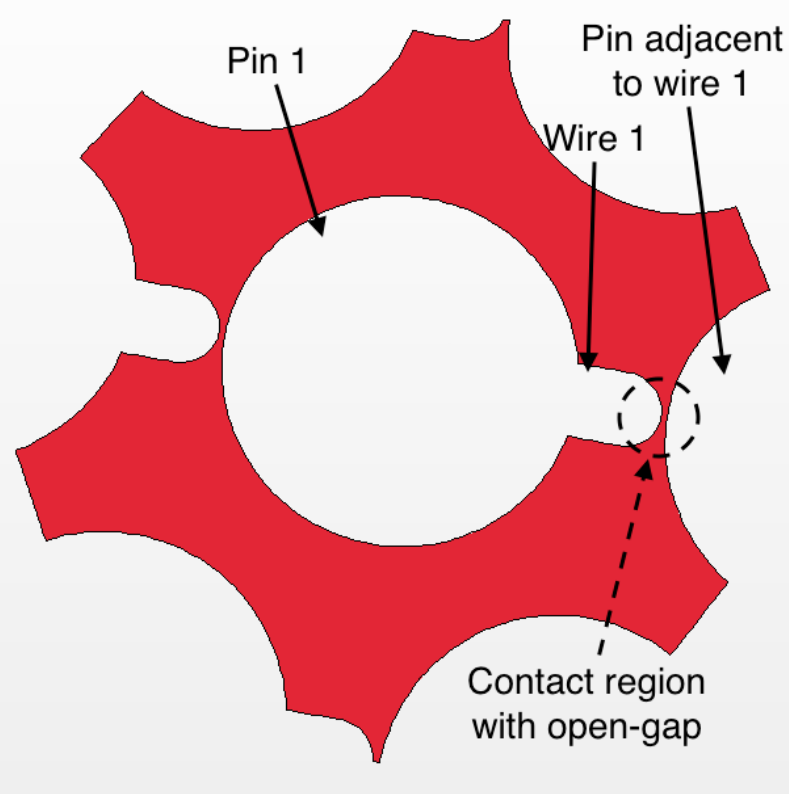

(b) Open-gap appraoch.

Figure 3. closed-gap approach versus open-gap approach for the single-pin bundle geometry.

\section{TURBULENT MODELS}

Once the geometry is meshed, the mesh is imported to STAR-CCM+ and the problem must be set, which requires choosing a turbulent model. For a given geometry and mesh, the choice of turbulent model can influence the predicted pressure drop. Consequently, as part of this study, sensitivity analysis to three turbulent models is proposed:

1. Realizable $k-\epsilon$ two-layer turbulent model (default $k-\epsilon$ turbulent model)

2. V2F variation of the $k-\epsilon$ turbulent model

3. The shear stress transport (SST) $k-\omega$ turbulent model with default settings

The Realizable $k-\epsilon$ turbulent model is an improvement over the standard $k-\epsilon$ model that is in theory unsuitable for applications in the viscous sublayer. The V2F variation of the $\mathrm{k}$ - model was designed to improve the accuracy of the $k-\omega$ model near walls and thus to better capture near-wall turbulent effects. The third turbulent model of interest in the well-established SST $k-\omega$ model, which is capable of resolving boundary layers. 


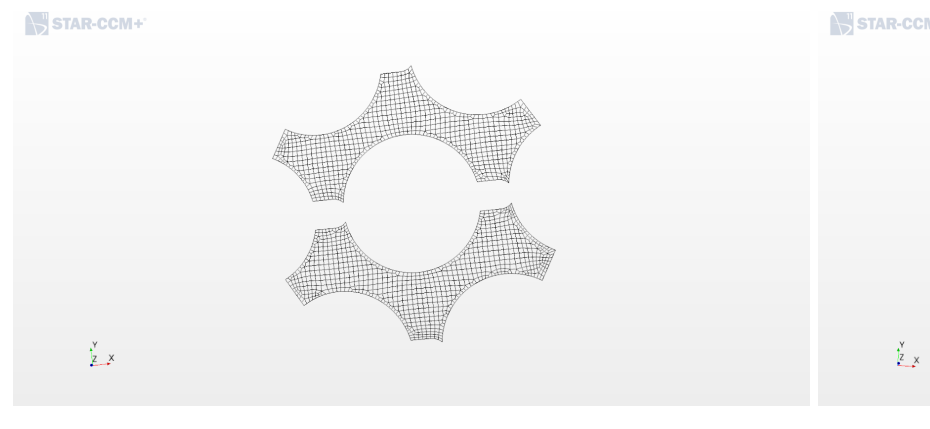

(a) closed-gap hex mesh.

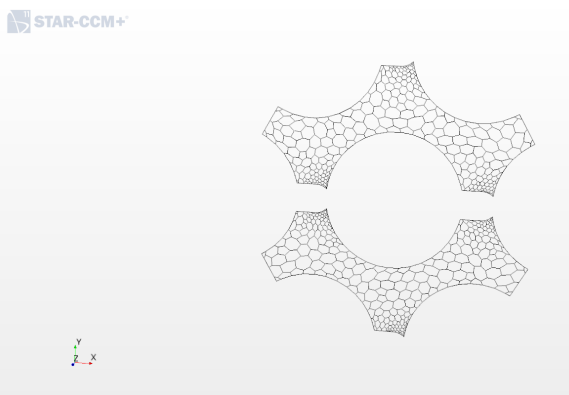

(c) closed-gap poly mesh.

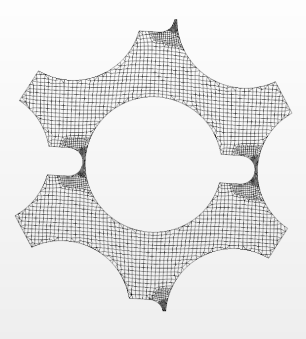

(b) Open-gap hex mesh.

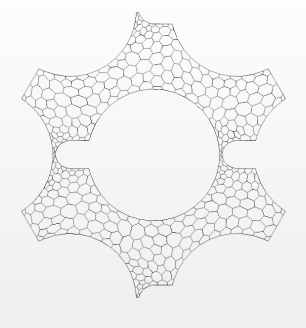

(d) Open-gap poly mesh.

Figure 4. Hex and poly meshes of the single-pin geometry for closed-gap approach and open-gap approach.

\section{THE SINGLE-PIN GEOMETRY: NUMERICAL RESULTS AND SENSITIVITY ANALYSIS}

This section presents numerical results obtained for the single-pin geometry, along with a sensitivity analysis of the pressure drop to the mesh density, the mesh type, the contact region model, and the three turbulent models chosen in Section 6. This section starts with some details regarding the model, boundary conditions and initial conditions, used to obtain the numerical results with the STAR-CCM+ CFD package.

\subsection{Boundary Conditions, Initial Conditions and Workflow}

The THORS facility collected pressure drop data for isothermal and heated flow tests at a subchannel Reynolds number ranging from $35 \times 10^{3}$ to $350 \times 10^{3}$. It is proposed to numerically predict pressure drops of isothermal flows in the single-pin geometry modeled with the closed- and open-gap approaches described in Section 5. using RANS models available in the STAR-CCM+ CFD package [4]. For each geometry modeled with the two closed- and open-gap approaches, three turbulent models and 15 inlet Reynolds numbers are considered, yielding a total of 90 STAR-CCM+ runs. Because of the large number of simulations, the choice was made to run STAR-CCM+ with the Dakota package [1] that allows for easy collection of statics with respect to the input parameters.

Inlet and outlet conditions are imposed at the boundaries in the stream-wise direction in all geometries. The single-pin bundle requires periodic boundary conditions in the span-wise directions: this approach is equivalent to simulating an infinite lattice. Wall boundary conditions are applied to pins and wires. Each geometry was meshed with polyhedral elements without the boundary layer option. The segregated flow 

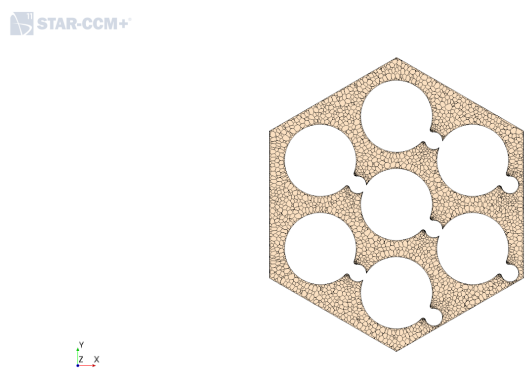

(a) closed-gap approach.

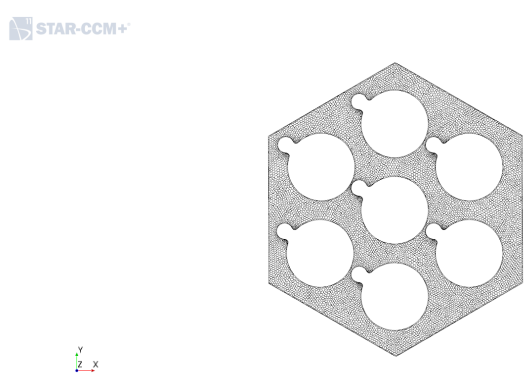

(b) Open-gap approach.

Figure 5. closed-gap approach versus open-gap approach for the 7-pin bundle geometry.

solver was used, as it is the default solver when using RANS turbulent models in STAR-CCM+. Each simulation was run on 32 processors until convergence criteria on the continuity equation, the momentum equations, and the turbulent residuals were met. Once the simulation was converged, the pressure drop was extracted and returned to Dakota for post-processing.

\subsection{Sensitivity Analysis of the Pressure Drop to the Mesh Density}

A sensitivity analysis was performed to determine the effects of the mesh density on the pressure drop for the single-pin geometry when meshed with polyhedral elements. Four different meshes were investigated for each model of the contact region and for each turbulent model, yielding a total of 24 runs. Once a steady solution was obtained, the pressure drop was recovered and is presented in Table 2. As the mesh

Table 2. Sensitivity of pressure drop to polyhedral mesh density for the single-pin geometry

\begin{tabular}{llll}
$\begin{array}{l}\text { turbulent } \\
\text { model }\end{array}$ & $\begin{array}{l}\text { contact } \\
\text { model }\end{array}$ & $\begin{array}{l}\text { number of } \\
\text { elements }\left(10^{3}\right)\end{array}$ & $\begin{array}{l}\text { pressure } \\
\text { drop }(\mathrm{kPa})\end{array}$ \\
1 & open-gap & $36,136,287,619$ & $47.5,45.8,44.9,44.5$ \\
2 & open-gap & $36,136,287,619$ & $47.2,45.4,44.5,44.1$ \\
3 & open-gap & $36,136,287,619$ & $47.3,45.8,45.2,45.0$ \\
1 & closed-gap & $196,334,796,1.252$ & $39.6,39.0,38.7,38.7$ \\
2 & closed-gap & $196,334,796,1.252$ & $39.5,38.9,38.6,38.5$ \\
3 & closed-gap & $196,334,796,1.252$ & $39.8,39.4,39.1,39.3$ \\
\hline
\end{tabular}

was refined for the open-gap approach, the pressure drop decreases to reach a converged value of $44.4 \mathrm{kPa}$ for turbulent model 1, $44.1 \mathrm{kPa}$ for turbulent model 2, and $45.0 \mathrm{kPa}$ for turbulent model 3 . The same pattern is observed for the closed-gap approach when the mesh is refined: the pressure drop decreases to reach values of $38.7 \mathrm{kPa}, 38.5 \mathrm{kPa}$ and $39.3 \mathrm{kPa}$ for turbulent models 1,2 , and 3 , respectively. Pressure drop results given in Table 2 clearly show that a polyhedral mesh of 287,000 ( $s p b$ ) elements for the open-gap approach and a polyhedral mesh of 796,000 elements (spa) for the closed-gap approach are fine enough to resolve the pressure drop for all considered turbulent models. In the remainder of this report, all numerical results presented for the single-pin geometry with a polyhedral mesh will be run with the meshes denoted by $s p a$ and $s p b$. Differences between the pressure drop obtained between the closed-gap approach 
and the open-gap approach are discussed in Section 7.6.

\subsection{Sensitivity Analysis of the Pressure Drop to Turbulent Models}

The pressure drop predicted by STAR-CCM+ is now investigated as a function of the turbulent models 1, 2 and 3 for both hexahedral and polyhedral meshes, and the closed-gap and open-gap approaches. Results are presented in Table 3, along with the pressure drop computed from the Cheng-Todreas correlation.

Table 3. Sensitivity of pressure drop to turbulent models for the single-pin geometry

\begin{tabular}{lllll}
\hline turbulent & contact & number of & pressure & Cheng-Todreas \\
model & model & elements $\left(10^{3}\right)$ & drop $(\mathrm{kPa})$ & correlation $(\mathrm{kPa})$ \\
1 & open-gap & hex - poly & $37.2-44.9$ & 40.1 \\
2 & open-gap & hex - poly & $35.6-44.5$ & 40.1 \\
3 & open-gap & hex - poly & $38.7-45.2$ & 40.1 \\
1 & closed-gap & hex - poly & $39.6-38.7$ & 40.1 \\
2 & closed-gap & hex - poly & $38.9-38.6$ & 40.1 \\
3 & closed-gap & hex - poly & $40.8-39.1$ & 40.1 \\
\hline
\end{tabular}

For the open-gap case, the predicted pressure drop with STAR-CCM+ is not very sensitive to the turbulent model, but it is very sensitive to the mesh type, with a pressure difference between $6.5 \mathrm{kPa}$ and $9.9 \mathrm{kPa}$. When considering the closed-gap approach, the pressure drops are all contained within $2.2 \mathrm{kPa}$ and are closer to the pressure drop computed from Cheng-Todreas correlation. The best match with the Cheng-Todreas correlation is obtained for the single-pin geometry modeled with the closed-gap approach and run with turbulent model 3.

\subsection{Sensitivity Analysis of Pressure Drop to Mesh Type}

A sensitivity analysis of the pressure drop to the mesh type is now performed with only turbulent model 3 . A single-pin geometry is modeled with the closed-gap approach and the open-gap-approach and meshed with polyhedral and hexahedral elements. Once a steady and converged solution is obtained, the pressure drop is extracted as shown in Table 4. The open-gap approach seems to be more sensitive to the mesh type

Table 4. Sensitivity of pressure drop to mesh type (hex and poly) for the single-pin geometry

\begin{tabular}{lllll}
\hline turbulent & contact & mesh & pressure & pressure drop \\
model & model & type & drop $(\mathrm{kPa})$ & difference $(\mathrm{kPa})$ \\
3 & open-gap & hex (sha) - poly $(\mathrm{spb})$ & $38.7-45.2$ & 6.5 \\
3 & closed-gap & hex (sha) - poly $(\mathrm{spb})$ & $40.8-39.1$ & 1.7 \\
\hline
\end{tabular}

than the closed-gap approach, with a pressure drop difference of $6.5 \mathrm{kPa}$ between hex and poly meshes. In the closed-gap approach, the pressure drop values are much closer with a pressure drop difference of 1.7 $\mathrm{kPa}$ between the polyhedral and hexahedral meshes. It is also observed that the pressure drop obtained from the two hexahedral meshes sha and $h s b$, and the polyhedral mesh with closed-gap approach, yield pressure drops within a pressure difference of $2.1 \mathrm{kPa}$. 


\subsection{Sensitivity Analysis of the Pressure Drop to the Number of Wire Pitch}

In the published literature, pressure drop simulations of wire-wrapped geometries are often performed on a single wire-pitch to limit computing resources. The validity of this assumption was assessed by simulating the pressure drop of a single-pin geometry over two wire-pitch, and the predicted value was compared against the pressure drop over a single-pitch. This study was limited to turbulent model 3 and to the single-pin geometry meshed with polyhedral elements. The single-pin geometries with two wire-pitches were obtained by duplicating the original geometry inside the STAR-CCM+ CFD package, and by creating an interface between the two geometries. The mesh was unchanged. Both the open-gap approach and the closed-gap approach were considered in this study. Results are presented in Table 5.

Table 5. Sensitivity of pressure drop to number of lead wire-pitch for the single-pin geometry

\begin{tabular}{lllll}
\hline turbulent & contact & number of & pressure & pressure drop \\
model & model & lead wire-pitch & drop $(\mathrm{kPa})$ & per unit pitch \\
3 & open-gap & $1-2$ & $45.2-88.0$ & $45.2-44.0$ \\
3 & closed-gap & $1-2$ & $39.1-77.1$ & $39.1-38.5$ \\
\hline
\end{tabular}

In the open-gap approach, the pressure drop per unit pitch differed by $1.2 \mathrm{kPa}$ between the single-wire pitch geometry and the two-wire pitch geometry. The same conclusion was drawn for the closed-gap approach, but with a pressure drop difference of $0.6 \mathrm{kPa}$. For the closed-gap and the open-gap approaches, the pressure drop predicted by STAR-CCM+ with one wire-pitch was accurate enough and thus enhanced the initial assumption of simulating a single wire-pitch for pressure drop calculations.

\subsection{Comparison of STAR-CCM+ Pressure Drops with Experimental and Correlation Data}

The numerical pressure drops obtained with the RANS models implemented in STAR-CCM+ are compared against experimental data collected for the THORS geometry. Pressure drop numerical results are plotted against data from the Cheng-Todreas correlation and experimental data for Reynolds number ranging from $8 \times 10^{3}$ to $350 \times 10^{3}$. The relative errors between the STAR-CCM+ pressure drops and the experimental data are also provided, along with the relative error between the STAR-CCM+ results and the Cheng-Todreas correlation. Results are shown in Figs. 6, 7, 8 and 9 for the polyhedral and hexahedral meshes with open- and closed-gap approaches. The first conclusion drawn from the pressure drop plots is that the hexahedral meshes yield the best match to the Cheng-Todreas correlation values. The polyhedral meshes overestimate the correlation data with the open-gap approach and underestimate them with the closed-gap approach. This effect becomes more obvious as the Reynolds number increases. From the relative error plots, it is observed that the hexahedral and polyhedral meshes match the experimental data within a $40 \%$ error for all Reynolds numbers. Two regions can be distinguished. In the region of Reynolds numbers inferior to $10^{5}$, the error with respect to the experimental data is inferior to $20 \%$. For Reynolds numbers higher than $10^{5}$ (i.e., the second region) the error is of the order on $40 \%$ for the closed-gap approach and on $60 \%$ for the open-gap approach, independently of the mesh type. The three turbulent models yields the comparable relative errors for all meshes and all Reynolds numbers. The SST turbulent model, however, yields the best estimate of the pressure drop when compared to the Cheng-Todreas correlations for the hexagonal meshes only. 


\section{THE 7-PIN WIRE-WRAPPED GEOMETRY: NUMERICAL RESULTS AND SENSITIVITY ANALYSIS}

This section presents a sensitivity analysis of the pressure drop to the turbulent models, the open-gap approach, and the closed-gap approach.

\subsection{Boundary Conditions, Initial Conditions and Workflow}

An isothermal flow in a THORS 7-pin wire-wrapped bundle geometry was simulated with the STAR-CCM+ CFD package using an incompressible model. Inlet and outlet boundary conditions were applied to the ends of the geometry, while wall boundary conditions were used for the shell surrounding the pins. The meshes are only composed of polyhedral elements $7 \mathrm{a}$ and $7 \mathrm{~b}$ (see Table 1 ). The workflow was identical to that used for the study of the single-pin geometry: the Dakota package was used to drive the simulations to run and to collect the pressure drop once the solution was converged for post-processing.

\subsection{Sensitivity of the Pressure Drops to the Open- and Closed-gap Approaches}

The pressure drops obtained with the RANS models implemented in STAR-CCM+ were plotted as a function of the Reynolds numbers, along with Cheng-Todreas correlation pressure drops and experimental data from the THORS facility. The relative error between the STAR-CCM+ pressure drops and the experimental data was also given. All results are presented in Fig. 10 and Fig. 11 for the 7-pin geometry meshed with polyhedral elements and modeled with the open- and closed-gap approaches.

The relative error plots between STAR-CCM+ results and the experimental data are given in Fig. 10b, and Fig. $11 \mathrm{~b}$ shows that the pressure drop obtained with the open-gap approach matches the experimental data better. Two regions are distinguished. In the low Reynolds number region $\left(\operatorname{Re}<10^{5}\right)$, the relative error is lower than in the high Reynolds number region, where the relative error reaches a plateau value of $25 \%$ for the open-gap approach and $60 \%$ for the closed-gap approach.

The relative error between STAR-CCM+ pressure drops and the Cheng-Todreas correlation are presented in Figs. 10c and 11c for the closed- and open-gap approaches, respectively. Whereas the relative error decreases from $100 \%$ to below $30 \%$ with the Reynolds number for the closed-gap approach, the relative error remains below $25 \%$ for the open-gap approach. The open-gap approach yields better results independently of the Reynolds number when compared to the Cheng-Todreas correlation, and thus should be favored. 


\section{THE 19-PIN WIRE-WRAPPED GEOMETRY: NUMERICAL RESULTS AND SENSITIVITY ANALYSIS}

An isothermal flow in a THORS 19-pin wire-wrapped bundle geometry was simulated with the STAR-CCM+ CFD package using the same workflow, procedure, and parameters as for the 7-pin geometry presented in Section 8.. Only the open-gap approach was investigated, but further work will be performed with the closed-gap approach. The pressure drops obtained with STAR-CCM+ are presented and compared to the THORS experimental data and the Cheng-Todreas correlation data in Fig. 12. In Fig. 12b, the relative error between STAR-CCM+ results and the experimental data show that better accuracy is achieved than for the 7-pin geometry. This is expected as the THORS experimental data were obtained with a 19-pin bundle. On the other hand, in the relative error plot shown in Fig. 12c between STAR-CCM+ pressure drops and the Cheng-Todreas correlation data show that better accuracy is achieved as the Reynolds number increases. 


\section{Conclusions}

A sensitivity analysis was performed on the modeling of the wire-adjacent pin contact region by considering three geometries: a single subchannel wire-wrapped bundle, a 7-pin wire -wrapped bundle, and a 19-pin wire-wrapped bundle. The sensitivity analysis study of the single-subchannel geometry highlighted the effect of the mesh type, the turbulent models, and the open- and closed-gap approaches. From the results obtained with STAR-CCM+, it is concluded that in the case of the single-subchannel geometry, the best match to the Cheng-Todreas correlation data and the experimental data is obtained with the combination of the hexahedral mesh, the open-gap approach, and the SST turbulent model. This conclusion was confirmed by the study of the velocity magnitude profile and velocity fields. The velocity fields obtained with the polyhedral meshes are not consistent with the periodic boundary conditions applied in the span-wire directions.

In the case of the 7-pin geometry, the polyhedral element mesh was chosen instead of the hexahedral element mesh to ease the computational effort. Polyhedral meshes are also known to yield better results when conjugate heat transfer is considered (not done in this study). The study of the pressure drop values obtained with STAR-CCM+ showed that when the open-gap approach is run with the SST turbulent model, it yields numerical results that better match the experimental data. The velocity fields show that the flow rotates and the velocity magnitude profiles display axial velocity peaks. These results are consistent with numerical results published in the open literature for the closed- and open-gap approaches. This study suggests that the extra computational effort required by the open-gap approach is required to accurately predict the pressure drop and to match experimental data within an acceptable tolerance. It is also noted that the SST turbulent models yield better results.

Results from the 19-pin geometry with the open-gap approach showed inconclusive results. Even though the 19-pin geometry with open-gap approach match the experimental data better than the 7-pin geometry, the relative error between the STAR-CCM+ results and the Cheng-Todreas correlation displayed a behavior that to that observed for the 7-pin geometry modeled with the closed-gap approach, i.e., the relative error decreases with the Reynolds number.

This sensitivity analysis will be completed in the near future by simulating the flow in a 19-pin wire-wrapped geometry with the closed-gap approach. The geometry will be meshed with polyhedral elements to minimize the mesh size and the computational effort. This also precedes future sensitivity analysis studies that will involve conjugate heat transfer, which is known to be handled better by polyhedral elements. Hexahedral element meshes are too large to be considered for 19-pin geometry. The objective of this study is to draw a clear conclusion on whether or not the approach used to model the contact point between the wire and the adjacent pin has a significant effect on the numerical prediction of the pressure drop in a wire-wrapped pin bundle. 


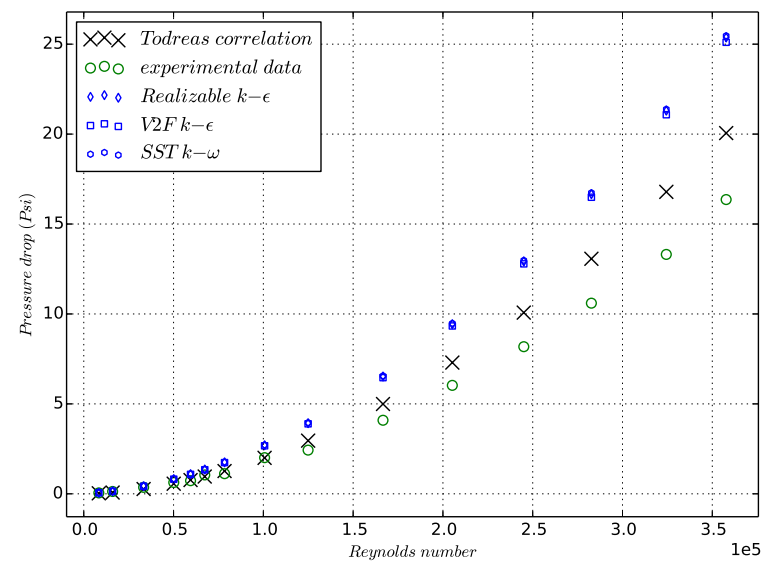

(a) Plots of the pressure drops as a function of subchannel Reynolds numbers.

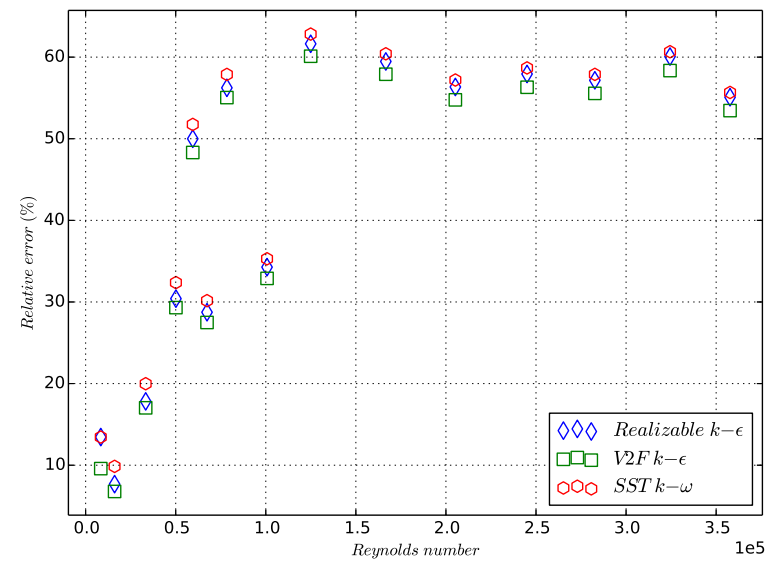

(b) Relative error between STAR-CCM+ results and experimental data.

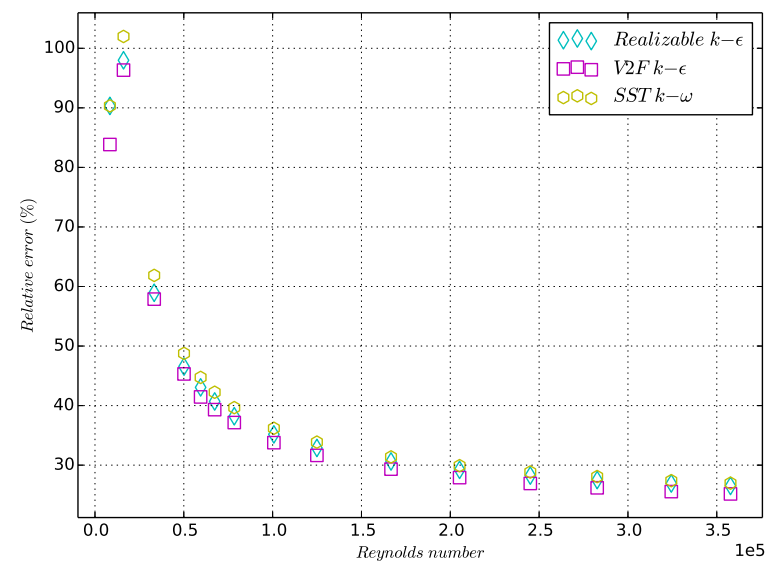

(c) Relative error between STAR-CCM+ results and correlation data.

Figure 6. Comparison of STAR-CCM+ pressure drops between experimental and correlation data with the polyhedral mesh and open-gap approach for the single-pin geometry. 


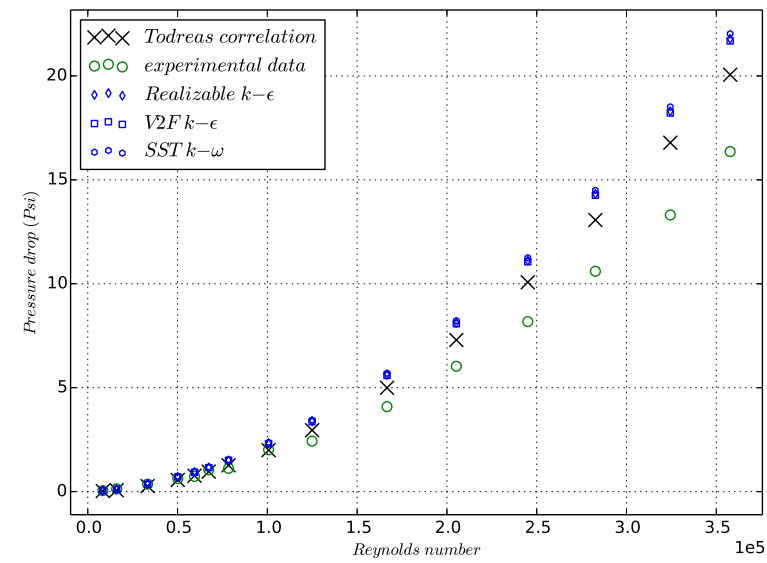

(a) Plots of the pressure drops as a function of subchannel Reynolds numbers.

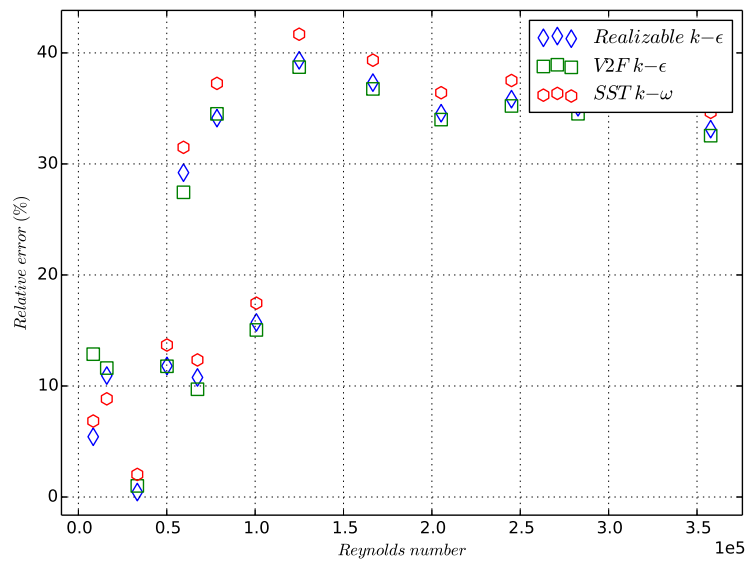

(b) Relative error between STAR-CCM+ results and experimental data.

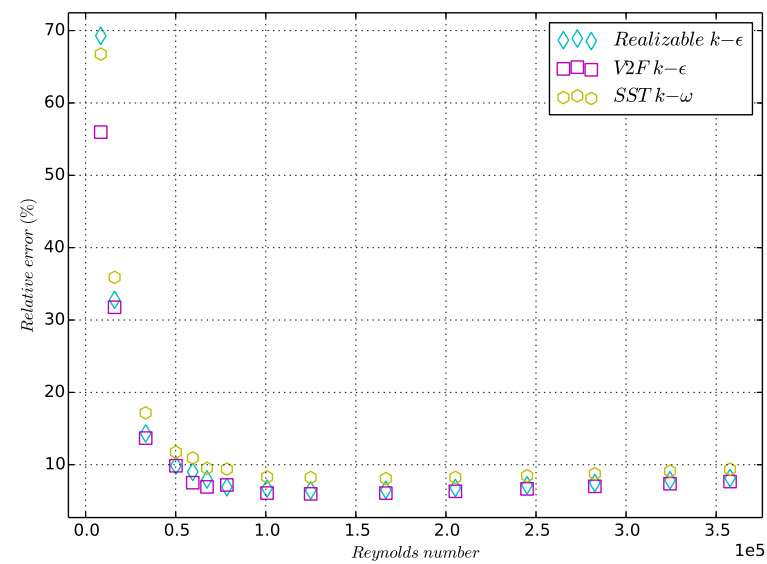

(c) Relative error between STAR-CCM+ results and correlation data.

Figure 7. Comparison of STAR-CCM+ pressure drops between experimental and correlation data with the polyhedral mesh and closed-gap approach for single-pin geometry. 


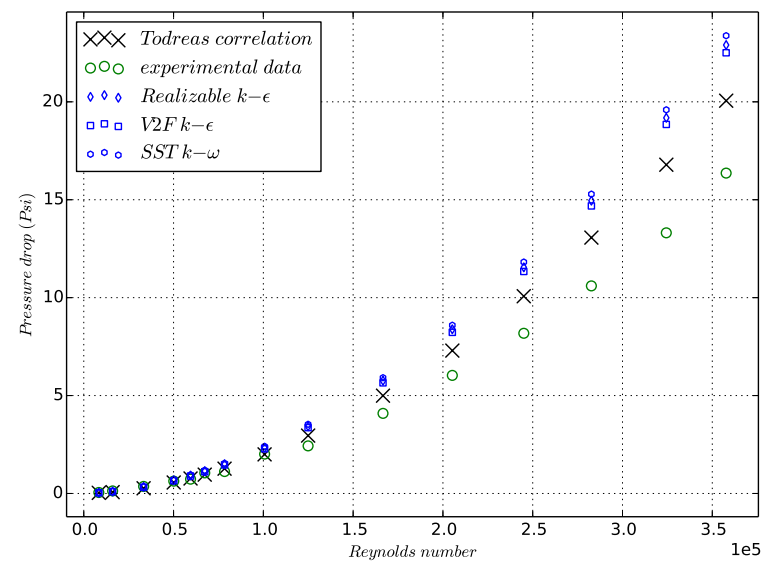

(a) Plots of the pressure drops as a function of subchannel Reynolds numbers.

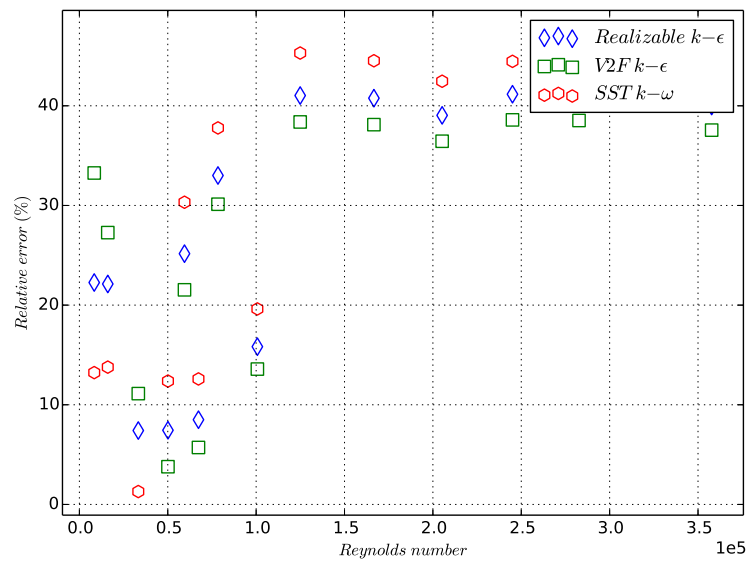

(b) Relative error between STAR-CCM+ results and experimental data.

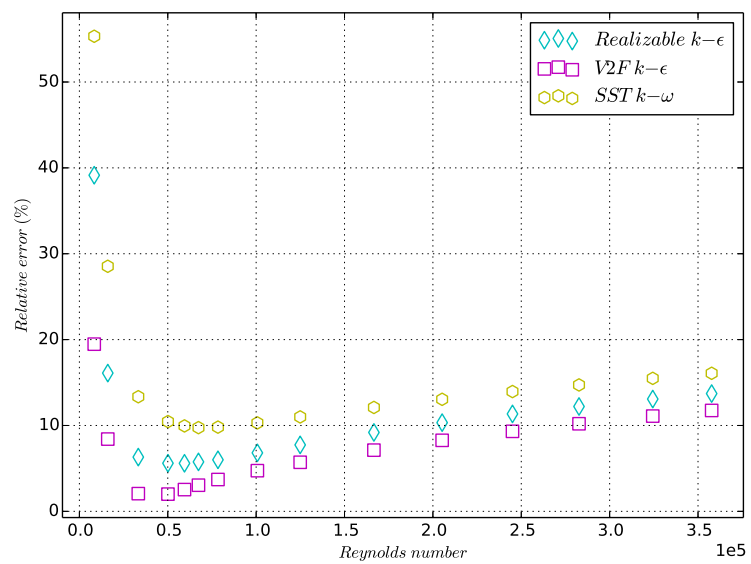

(c) Relative error between STAR-CCM+ results and correlation data.

Figure 8. Comparison of STAR-CCM+ pressure drops between experimental and correlation data with the hexahedral mesh and closed-gap approach for the single-pin geometry. 


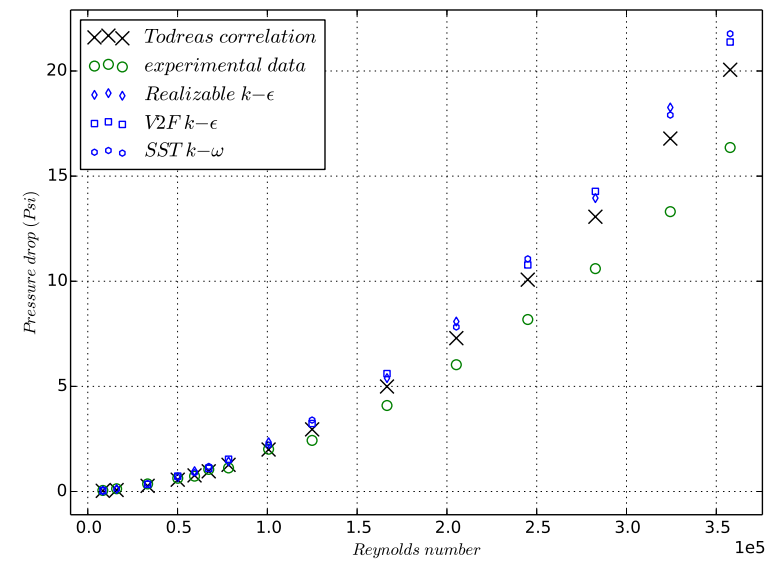

(a) Plots of the pressure drops as a function of subchannel Reynolds numbers.

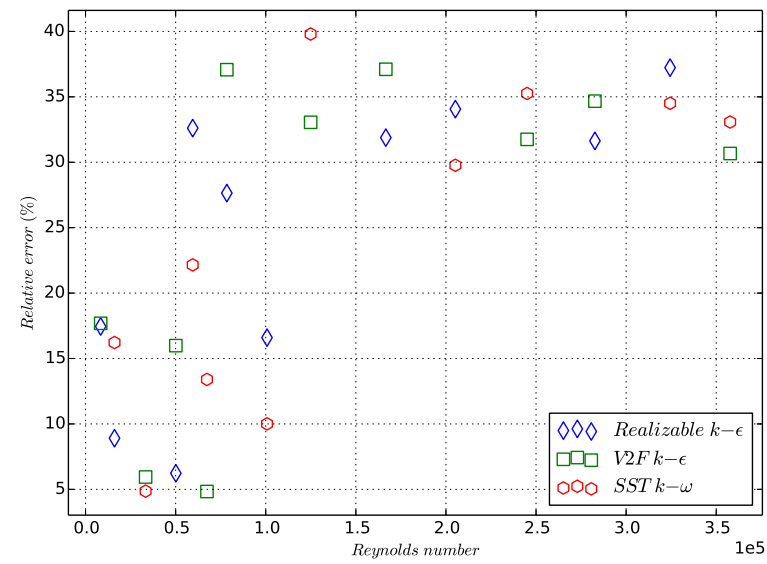

(b) Relative error between STAR-CCM+ results and experimental data.

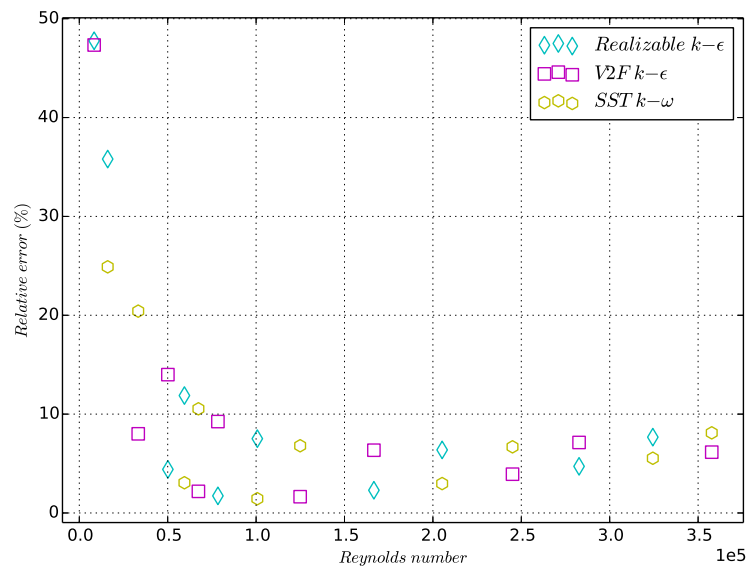

(c) Relative error between STAR-CCM+ results and correlation data.

Figure 9. Comparison of STAR-CCM+ pressure drops between experimental and correlation data with the hexahedral mesh and open-gap approach for the single-pin geometry. 


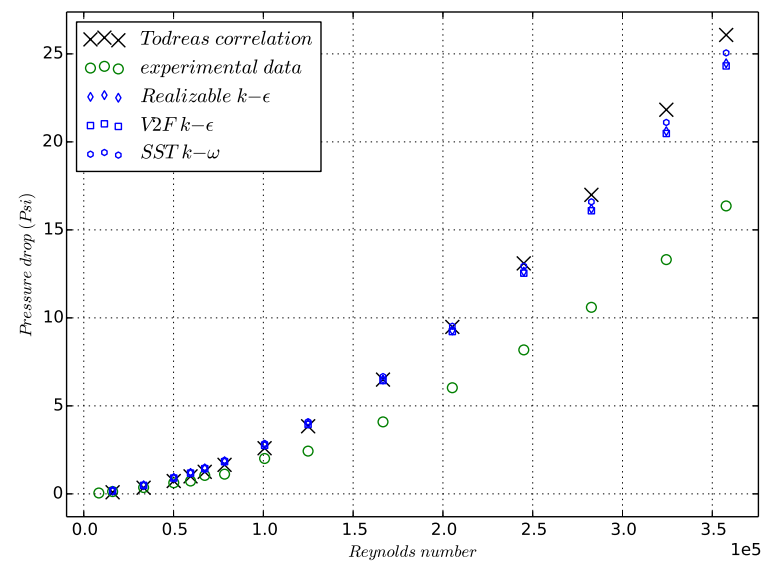

(a) Plots of the pressure drops as a function of subchannel Reynolds numbers.

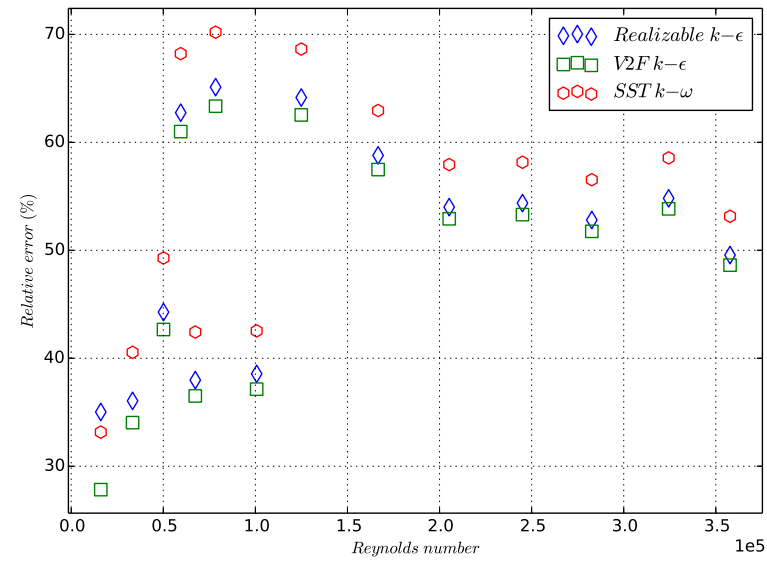

(b) Relative error between STAR-CCM+ results and experimental data.

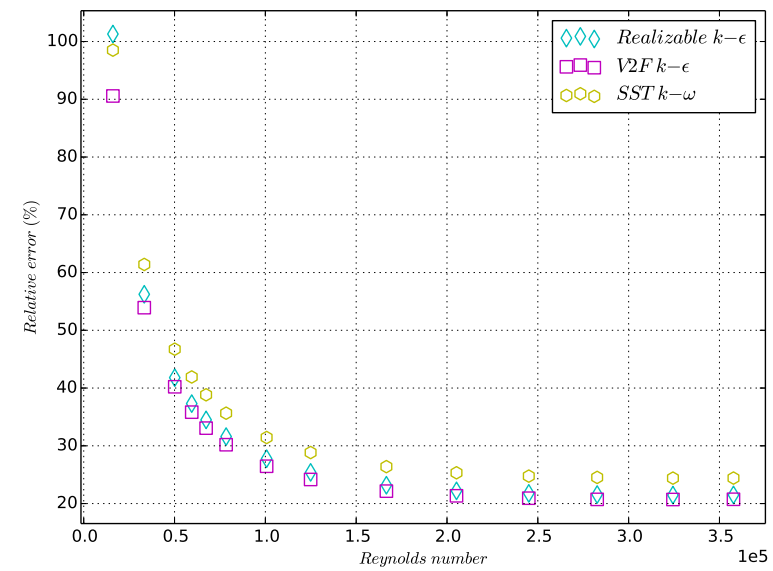

(c) Relative error between STAR-CCM+ results and correlation data.

Figure 10. Comparison of STAR-CCM+ pressure drop results between experimental and correlation data for the 7-pin geometry with closed-gap approach and polyhedral mesh. 


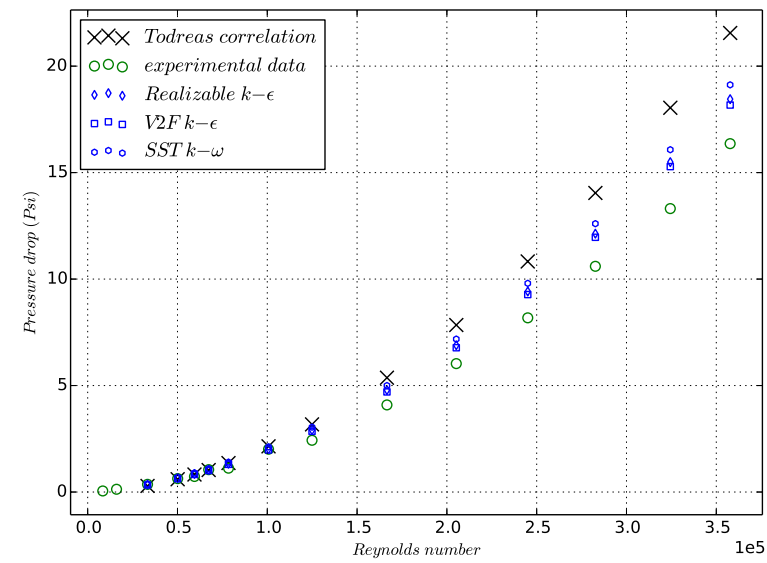

(a) Plots of the pressure drops as a function of subchannel Reynolds numbers.

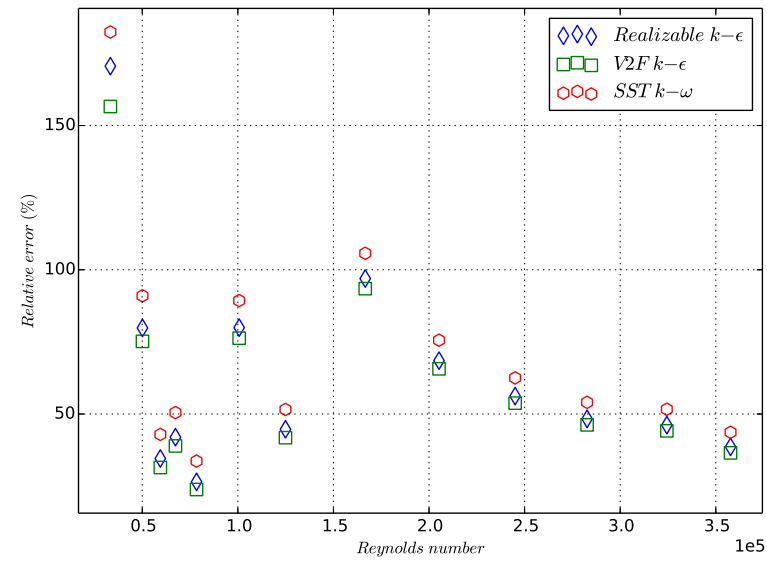

(b) Relative error between STAR-CCM+ results and experimental data.

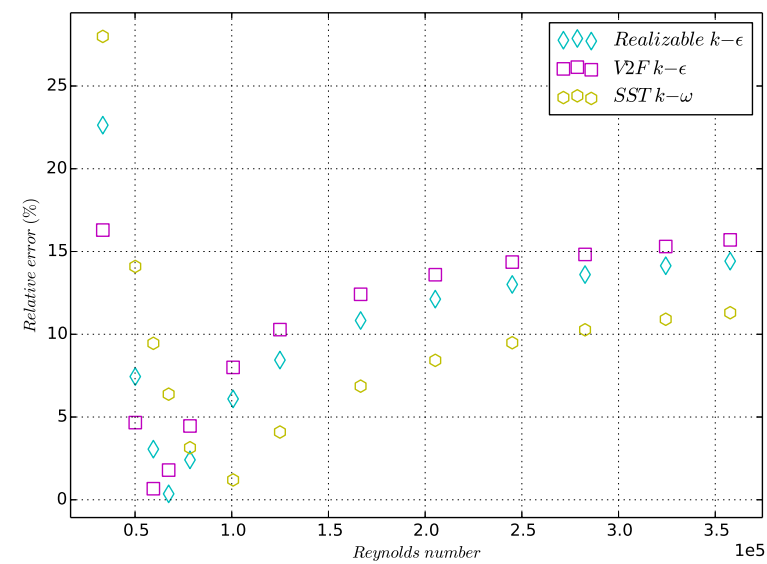

(c) Relative error between STAR-CCM+ results and correlation data.

Figure 11. Comparison of STAR-CCM+ pressure drop results between experimental and correlation data for the 7-pin geometry with open-gap approach and polyhedral mesh. 


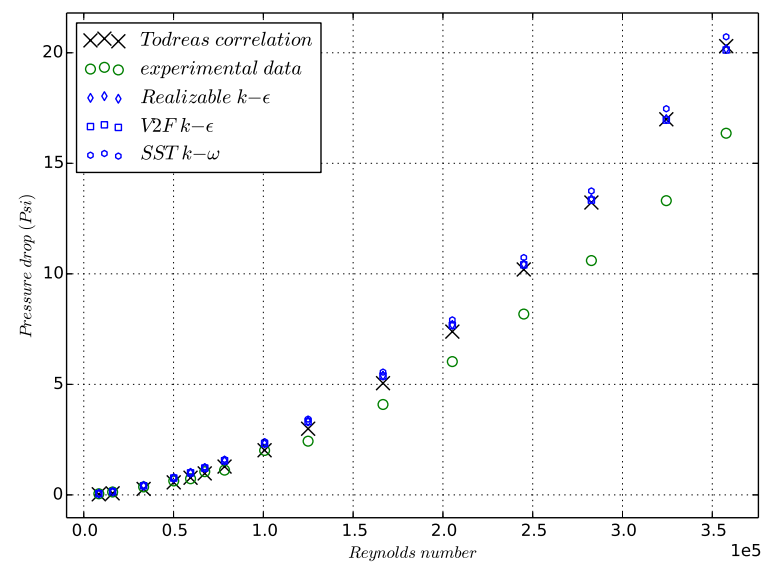

(a) Plots of the pressure drops as a function of subchannel Reynolds numbers.

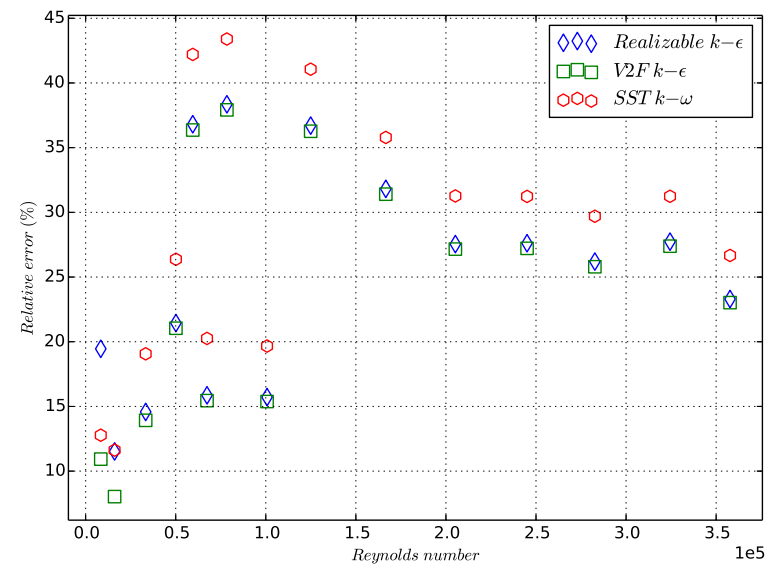

(b) Relative error between STAR-CCM+ results and experimental data.

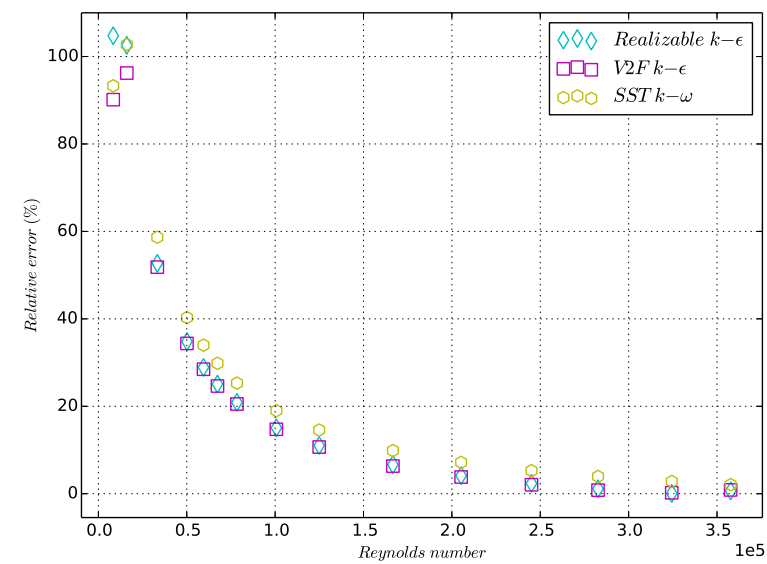

(c) Relative error between STAR-CCM+ results and correlation data.

Figure 12. Comparison of STAR-CCM+ pressure drop results between experimental and correlation data for the 19-pin geometry with open-gap approach and polyhedral mesh. 



\section{REFERENCES}

\section{References}

[1] B. M. Adams, L. E. Bauman, W. J. Bohnhoff, K. R. Dalbey, M. S. Ebeida, J. P. Eddy, M. S. Eldred, P. D. Hough, K. T. Hu, J. D. Jakeman, J. A. Stephens, L. P. Swiler, D. M. Vigil, and T. M. Wildey. Dakota, A Multilevel Parallel Object-Oriented Framework for Design Optimization, Parameter Estimation, Uncertainty Quantification, and Sensitivity Analysis: Version 6.0 User s Manual. Technical report, Sandia National Laboratory SAND2014-4633, July 2014.

[2] A. Ahmad and K.-Y. Kim. "Three-dimensional analysis of flow and heat transfer in a wire-wrapped fuel assembly". In Proc. of ICAPP 2005, Seoul, Korea, 2005.

[3] J. Carbajo, E. Popov, and D. Pointer. "Review of legacy THORS fuel bundle experiments as validation basis for NEK5000 simulations of wire-wrapped sodium-cooled fast reactor (SFR).". Technical Report ORNL-TM, Oak Ridge National Laboratory, 2016.

[4] CD-Adapco. Star-ccm+. http://www.cd-adapco.com/products/star-ccm, 2000.

[5] S. Chen, N. Todreas, and N. Nguyen. "Evaluation of existing correlations for the prediction of pressure drop in wire-wrapped hexagonal array pin bundles". Nuclear Engineering Design, 267:109-131, 2014.

[6] P. Fisher, J. Lottes, and A. Siegel. "Large eddy simulation of wire-wrapped fuel pins i: Hydrodynamics in a periodic array". In Joint International Topical Meeting on Mathematics $\mathcal{E}$ Computation and Supercomputing in Nuclear Applications, Monterey, CA, USA.

[7] J.-H. Jeong, J. Yoo, K.-L. Lee, and K.-S. Ha. "Three-dimensional flow phenomena in a wire-wrapped 37-pin fuel bundle for SFR”. Nucl Eng Technol, (47):523-533, 2015.

[8] J. C. Macdougall and J. N. Lillington. "The SABRE code for fuel rod cluster thermo-hydraulics". Nuclear Engineering Design, (82):171-190, 1984.

[9] H. Ninokata. ASFRE III: A Computer Program for Triangular Rod Array Thermo-Hydraulic Analysis of Fast Breeder Reactors. Technical Report PNC-N941, PNC, 1985.

[10] E. H. Novendstern. "Turbulent flow pressure drop model for fuel rod assemblies utilizing a helical wire-wrap spacer system. Nuclear Engineering and Design, 92:19-27, 1972.

[11] W. D. Pointer, P. Fisher, and A. Siegel. "RANS-based CFD simulations of wire-wrapped fast reactor fuel assemblies". In Proceedings of the 2008 International Congress on Advances in Nuclear Power Plants - ICAPP, Anaheim, CA, USA, 2008.

[12] W. D. Pointer, J. Smith, A. Siegel, and P. Fisher. "RANS simulations of turbulent diffusion in wire-wrapped sodium fast reactor fuel assemblies". Proceedings of International Conference on Fast Reactors and Related Fuel Cycles (FR09), (Kyoto, Japan), Nov. 2009.

[13] K. Rehme. "Pressure drop correlations for fuel element spacers". Nucl. Technol, (17):15-23, 1973.

[14] D. S. Rowe. COBRA III.: A Digital Computer Program for Steady State and Transient Thermal Hydraulic Analysis of Nuclear Fuel Elements. Technical Report BNWL-1695, Battle Pacific Northwest Laboratories, 2016. 
[15] N. Todreas and S. Cheng. "Hydrodynamic models and correlations for bare and wire-wrapped hexagonal rod bundles - bundle friction factors, subchannel friction factors and mixing parameters.". Nuclear Engineering and Design, 92:227-251, 1985. 